\title{
Modelling of Non-Uniform Prominence Slabs
}

\author{
P. Heinzel
}

Astronomical Institute, Academy of Sciences of the Czech Republic CZ25165 Ondřejov, Czech Republic

\author{
U. Anzer \\ Max-Planck-Institut für Astrophysik, Postfach 1523 D-85740 Garching, \\ Germany
}

\begin{abstract}
We present new slab models for quiescent prominences in which both the condition of magnetohydrostatic equilibrium as well as NLTE are fulfilled. Using the Kippenhahn-Schliiter model, a unique relation between the components of the magnetic field on one hand and the gas pressure and width on the other is established for a given uniform temperature. We construct a grid of such NLTE models and compare them with the set of isothermal-isobaric models of Gouttebroze et al. (1993).
\end{abstract}

\section{Introduction}

An up-to-day study of NLTE properties of prominences has been recently performed by Gouttebroze et al. (1993 - GHV). For each of their 140 isothermalisobaric models they calculated the emergent spectrum in various hydrogen lines and the Lyman continuum. They also derived correlations between prominence plasma parameters and the emitted radiation (Heinzel et al. 1994- HGV). These isobaric slabs, however, cannot be in static equilibrium with their surroundings since: a) the vertical pressure scale-height for prominence material is a factor 100 smaller than that of the corona, and b) all high-density prominences would require unreasonably large values of the coronal pressure to confine them in the horizontal direction. Therefore, magnetic fields are definitely needed to support and confine the prominence plasma. In order to demonstrate how physical and optical properties of non-uniform models compare to those for isobaric ones, we perform here NLTE calculations for Kippenhahn-Schlüter (K-S) type prominences with the gas pressure varying according to the magnetohydrostatic equilibrium.

\section{Pressure Equilibrium}

The simplest magnetic equilibrium configuration which one can consider is the isothermal K-S-like configuration (see Anzer 1995). Such 1D magnetic slab configurations can also be described in terms of the column-mass coordinate, $m$, 
as done by Heasley and Mihalas (1976). With

$$
d m=-\rho d x
$$

one gets the pressure variations inside the slab in the form

$$
p(m)=4 p_{c} \frac{m}{M}\left(1-\frac{m}{M}\right)+p_{0}
$$

where $p_{0}$ is the gas pressure at the outer boundary (coronal pressure) and $\rho$ is the plasma density. This formulation has the advantage that slabs with finite width which extend from $m=0$ to $m=M$ can be treated quite naturally; in addition, it is valid for arbitrary temperature structure. Moreover, the column-mass scale represents a standard coordinate system for radiative transfer calculations. The magnetic field components must then satisfy the relations

$$
p_{c}=\frac{B_{z 1}^{2}}{8 \pi}
$$

and

$$
M=\frac{B_{x} B_{z 1}}{2 \pi g}
$$

where $B_{z 1}$ represents the vertical field-component at the prominence surface, which is defined by $x=x_{1} . p_{c}$ is the central gas pressure (see Anzer 1995).

In the present investigation we want to compare physical and radiative properties of isobaric slabs with those of K-S type which have the same total column mass and the same mean gas pressure (in order to see clearly the effect of various pressure profiles, we consider here only isothermal slabs). We shall define the mean pressure by the relation

$$
\bar{p}=\frac{1}{M} \int_{0}^{M} p d m,
$$

which gives

$$
\bar{p}=\frac{2}{3} p_{c}+p_{0}
$$

The thickness of the slab, which is given by $D=2 x_{1}$, can also be obtained from (see Heasley and Mihalas 1976)

$$
D=\int_{0}^{M} \frac{d m}{\rho}
$$

once $\rho(m)$ has been calculated from the constructed NLTE model. For the comparison of isobaric and K-S type configurations we also evaluate mean values of the gas pressure, hydrogen density and electron density using Eq. (5). To compute the emission measure, we use here the definition

$$
E M=\int_{0}^{M} n_{e}^{2} d m / \rho
$$




\section{Statistical Equilibrium}

Both isobaric and K-S type models are assumed to satisfy the statistical equilibrium, i.e., the hydrogen excitation and ionization is computed consistently with the radiation fields in all relevant transitions (for description of our NLTE treatment see Heinzel 1995). We use a five-level plus continuum hydrogen model atom which is accurate enough to evaluate the ionization structure, gas density and the emergent radiation in the $\mathrm{L} \alpha, \mathrm{L} \beta, \mathrm{L}$-cont and $\mathrm{H} \alpha$ transitions. We use a depth-dependent PRD treatment of Lyman lines as in GHV. We evaluate both isobaric and K-S models with the same NLTE code which allows us to make internally consistent comparisons.

\section{Model Calculations}

We have performed NLTE calculations for different isobaric and K-S slab configurations with temperature $T=8000 \mathrm{~K}$, and microturbulent velocity $v_{t}=$ $5 \mathrm{~km} / \mathrm{sec}$ (used only for the line broadening). The mean gas pressure was set to be $\bar{p}=0.05,0.1,0.5 \mathrm{dyn} / \mathrm{cm}^{2}$ and $M$ was chosen in such a way as to give us the geometrical thickness $D=500$ and $5000 \mathrm{~km}$. Isobaric models with these parameters are those considered by GHV. To evaluate K-S models, we just replace the mean gas pressure by $p(m)$ according to Eq. (2), with $p_{0}=0.01 \mathrm{dyn} / \mathrm{cm}^{2}$ and with $p_{c}=3\left(\bar{p}-p_{0}\right) / 2$; the values of $\bar{p}$ and $M$ are then taken from the corresponding isobaric slab model.

\section{Results and Conclusions}

For each model we have computed the relative deviations (in \%) of mean values of all physical and optical quantities. The deviations between individual parameters are defined as

$$
\Delta[\%]=\frac{\operatorname{par}(K S)-\operatorname{par}(\text { isobar })}{\operatorname{par}(\text { isobar })} \times 100 .
$$

All these parameters as evaluated for the K-S configuration differ only slightly from those computed with corresponding isobaric models. The differences are typically smaller than $5 \%$. Only the mean density of the hydrogen first-level population differs by about $10 \%$, and also the intensity of the Lyman continuum at its head is rather different for models with high pressure and large width (but still not exceeding $16 \%$ ). Very important is the fact that the deviation of the emission measure, $E M$, is negligible. We demonstrate this in Figure 1 which compares the previously-found correlation of GHV and the newly computed one for K-S-type, non-uniform slabs. This result means that neither $E M$ nor the $\mathrm{H} \alpha$ integrated intensity are sensitive to the pressure (density) variations in K-S slabs. And since the mean electron densities agree quite well, one can also use the correlation curve of GHV or HGV for K-S-type models and get the mean electron density. Alternatively, for a given mean electron density, $D$ can be obtained from $E M$. However, this value of $D$ is smaller than that defined by Eq. (7). To be more consistent with isobaric models (and to get about the same $D$ ), one has to define $D$ for K-S models as $D=M / \bar{\rho}$. 
Finally, our analysis suggests an interesting possibility to derive the magnetic field which is needed for a K-S-type magnetic equilibrium directly from the mean value of gas pressure, $M$ and $p_{0}$, using the formulae of Section 2. $\bar{p}$ and $M$ have to be determined from spectral diagnostics, where one can use - to a high degree of accuracy - the simple isobaric models.

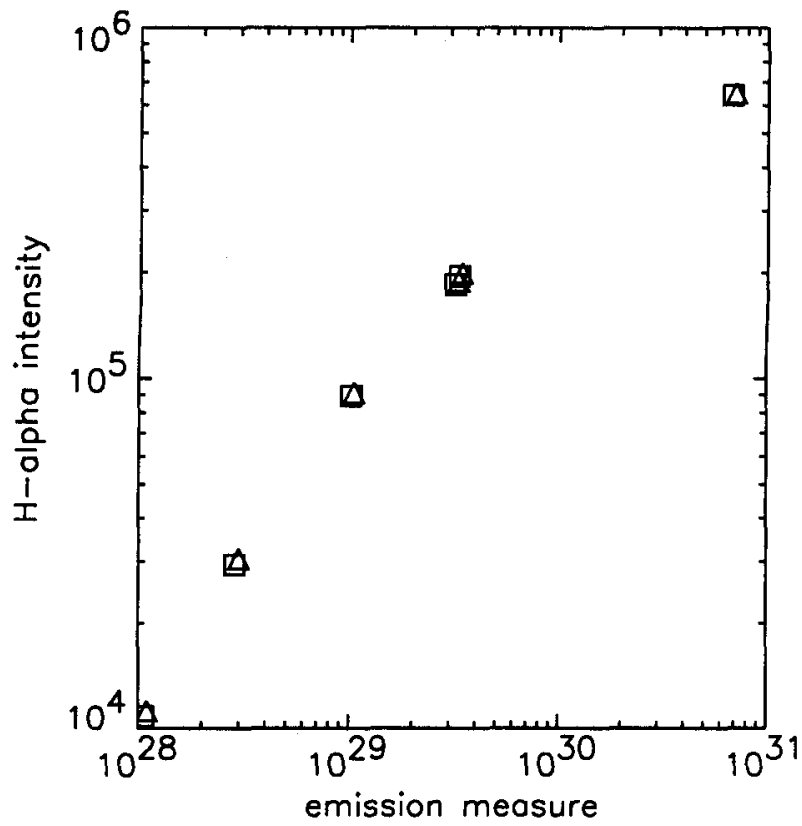

Figure 1. Integrated $\mathrm{H} \alpha$ intensity vs. emission measure $E M$ (in CGS units). Squares - K-S models, triangles - isobaric models.

\section{References}

Anzer, U. 1995, Solar Phys., 161, 49

Gouttebroze, P., Heinzel, P. and Vial, J.-C. 1993, A\&AS, 99, 513

Heasley, J.N. and Mihalas, D. 1976, ApJ, 205, 273

Heinzel, P. 1995, A\&A, 299, 563

Heinzel, P., Gouttebroze, P. and Vial, J.-C. 1994, A\&A, 292, 656 